\title{
Genotype and phenotype analyses in 136 patients with single large-scale mitochondrial DNA deletions
}

\author{
Shintaro Yamashita $\cdot$ Ichizo Nishino • \\ Ikuya Nonaka $\cdot$ Yu-ichi Goto
}

Received: 1 February 2008/Accepted: 17 March 2008/Published online: 15 April 2008

(c) The Japan Society of Human Genetics and Springer 2008

\begin{abstract}
We examined 136 patients with mitochondrial DNA (mtDNA) deletion. Clinical diagnoses included chronic progressive external ophthalmoplegia (94 patients); Kearns-Sayre syndrome (KSS; 33 patients); Pearson's marrow-pancreas syndrome (six patients); and Leigh syndrome, Reye-like syndrome, and mitochondrial myopathy, encephalopathy, lactic acidosis, and stroke-like episodes (one patient). The length and location of deletion were highly variable. Only one patient had deletion within the so-called shorter arc between the two origins of mtDNA replication. The length of deletion and the number of deleted transfer ribonucleic acid (tRNAs) showed a significant relationship with age at onset. Furthermore, KSS patients had longer and larger numbers of deleted tRNAs, which could be risk factors for the systemic involvement of single mtDNA deletion diseases. We found 81 patterns of deletion. Direct repeats of $4 \mathrm{bp}$ or longer flanking the breakpoints were found in 96 patients (70.5\%) and those of $10 \mathrm{bp}$ or longer in 49 patients $(36.0 \%)$. We found two other
\end{abstract}

S. Yamashita · Y. Goto $(\bowtie)$

Department of Mental Retardation and Birth Defect Research, National Institute of Neuroscience,

National Center of Neurology and Psychiatry (NCNP),

4-1-1 Ogawahigashi, Kodaira, Tokyo 187-8502, Japan

e-mail: goto@ncnp.go.jp

I. Nishino $\cdot$ I. Nonaka

Department of Neuromuscular Research,

National Institute of Neuroscience,

National Center of Neurology and Psychiatry (NCNP),

Kodaira, Tokyo, Japan

S. Yamashita

Department of Pediatrics and Adolescent Medicine,

Juntendo University School of Medicine,

Bunkyo-ku, Tokyo, Japan common deletions besides the most common deletion (34 patients: 25.0\%): the 2,310-bp deletion from nt 12113 to $\mathrm{nt}$ 14421 (11 patients: $8.0 \%$ ) and the 7,664-bp deletion from nt 6330 to nt 13993 (ten patients: 7.3\%). These deletions had incomplete direct repeats longer than $13 \mathrm{bp}$ with one base mismatch.

Keywords Mitochondrial DNA - Deletion - CPEO . KSS · Direct repeat

\section{Introduction}

Mitochondria are organelles found in every cell except in mature erythrocytes, and they function as an energy-converting factory. Mitochondrial dysfunction causes organ damage producing various clinical symptoms due to energy crisis in a cell. Nuclear gene and mitochondrial DNA (mtDNA) mutations are thought to be related to mitochondrial disease. MtDNA mutations are classified to depletion, deletion/duplication, and point mutations.

Deletions of the mtDNA were the first mutations reported in association with human disease (Holt et al. 1988). Some patients have duplications of mtDNA as well as deletions (Poulton et al. 1989). Subsequently, multiple mtDNA deletions have been described in familial cases (Zeviani et al. 1989; Servidei et al. 1991), and nuclear gene defects predisposing to multiple deletions have been documented (Nishino et al. 1999; Kaukonen et al. 2000; Van Goethem et al. 2001; Spelbrink et al. 2001).

Single large-scale deletions of mtDNA are typically associated with chronic progressive external ophthalmoplegia (CPEO), Kearns-Sayre syndrome (KSS), and Pearson's marrow-pancreas syndrome (PS) (Holt et al. 1988; Zeviani et al. 1988; Rötig et al. 1989; Campos et al. 
2000; Akman et al. 2004). Deletions exist in a heteroplasmic fashion, and the proportion of heteroplasmy is variable among cells and organs. Although a common 4,977-bp deletion is present (Schon et al. 1989), there appears to be many other deletions differing in length and position. Frequently, sequences at the breakpoints revealed the presence of direct repeats, suggesting the mechanism of deletion as slipped mispairing or homologous recombination (Shoffner et al. 1989; Mita et al. 1990).

In this study, we examined 136 Japanese patients with single large-scale mtDNA deletions for the clinical phenotypes and genomic characteristics of deletion, including the precise junction sequences to delineate genotype-phenotype correlation. Furthermore, we provided fundamental clinical information of the single large-scale mtDNA deletion disorder.

\section{Patients and methods}

\section{Patients}

Between 1987 and 2005, we examined 136 patients who had single large-scale deletions in the mtDNA obtained from the skeletal muscle or peripheral blood. Among 136 DNA samples, eight (six PS and two CPEO) were blood and the remaining 128 samples were all skeletal muscle. The result of muscle DNA was exclusively utilized to assess the relationship between the heteroplasmy and the age at onset. All patients gave informed consent before muscle biopsy or mtDNA analysis was performed. Clinical data, including clinical manifestations and laboratory findings, were obtained from the attending physicians at the time of muscle biopsy or mtDNA analysis. Some of the patients were previously described (Goto et al. 1990a, b; Nakai et al. 1994; Mori et al. 1991).

Detection of mtDNA deletions by Southern blot analysis, long polymerase chain reaction, and sequence determination of breakpoints

DNA samples from muscle biopsies were prepared by the method described previously (Goto et al. 1990a) and those from peripheral blood by the method of Miller et al. (1988). Southern blot analysis was performed according to the method described previously (Goto et al. 1990a). The proportion of the deleted mtDNA was measured by densitometrical analysis. Long polymerase chain reaction (PCR) amplification and junction sequence determination at breakpoints were performed as described previously (Goto et al. 1996).
Statistical analysis

To compare the quantitative parameters between two groups, we used the Mann-Whitney $U$ test. A $P$ value $<0.05$ was considered significant. We considered results of correlation coefficient as follows: between the range of \pm 0 and \pm 0.2 as no correlation, \pm 0.2 and \pm 0.4 as weak correlation, \pm 0.4 and \pm 0.7 as moderate correlation, and \pm 0.7 and \pm 1.0 as significant correlation.

\section{Results}

\section{Clinical diagnosis}

Sixty male and 76 female patients were included in the study. No family history was identified except in two patients who derived from the same family (Ozawa et al. 1988). Clinical diagnoses included CPEO in 94 patients (69\%), KSS in 33 (24\%), PS in six(4\%), and others in three (2\%). We adopted the criteria of KSS proposed by Rowland (1983). All KSS patients had ophthalmoplegia, pigmentary retinopathy, onset before age 20 , and one of the following: complete heart conduction block, high cerebrospinal fluid protein content, and cerebellar symptoms. Diagnosis of PS was made based on the presence or absence of anemia and/or vacuoles in erythroblasts or other hematologic cell lineages and pancreatic dysfunction. Major manifestations and selected laboratory findings of each clinical diagnosis are summarized in Table 1.

\section{Clinical manifestations and laboratory findings}

The incidence of major clinical manifestations and laboratory findings are summarized in Table 1. Ptosis/PEO was collectively found in $94 \%$ of patients, muscle weakness in $87 \%$, retinopathy in $34 \%$, heart conduction block in $24 \%$, deafness in $30 \%$, and short stature in $26 \%$. Major laboratory findings, including high $\mathrm{CK}$, high lactate levels in serum and cerebrospinal fluid (CSF), and increased CSF protein content, were detected in more than one third to almost one half of patients. Compared with CPEO, KSS had a high frequency of retinopathy, heart conduction block, and increased CSF protein content because of Rowland's criteria. However, it was notable that deafness $(64 \%)$ and short stature (70\%) were frequently observed in KSS, and insulin-dependent diabetes mellitus (IDDM) $(15 \%)$ and deToni-Fanconi-Debré syndrome (12\%) were sometimes prominent complications in KSS. Because we encountered only six PS patients, it was difficult to compare the incidence of major symptoms and laboratory findings with other phenotypes. 

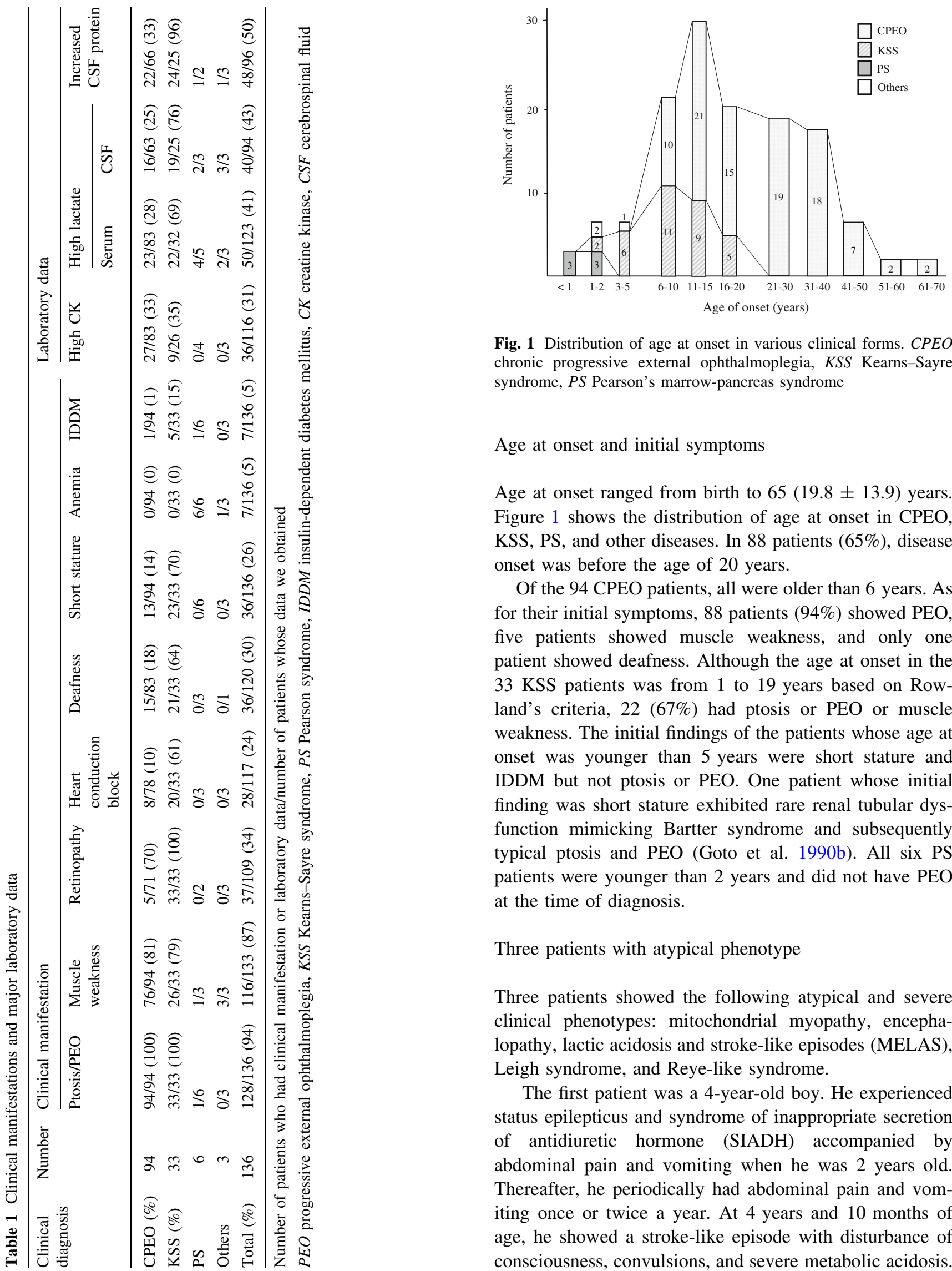

Fig. 1 Distribution of age at onset in various clinical forms. CPEO chronic progressive external ophthalmoplegia, KSS Kearns-Sayre syndrome, $P S$ Pearson's marrow-pancreas syndrome

Age at onset and initial symptoms

Age at onset ranged from birth to $65(19.8 \pm 13.9)$ years. Figure 1 shows the distribution of age at onset in CPEO, KSS, PS, and other diseases. In 88 patients (65\%), disease onset was before the age of 20 years.

Of the 94 CPEO patients, all were older than 6 years. As for their initial symptoms, 88 patients (94\%) showed PEO, five patients showed muscle weakness, and only one patient showed deafness. Although the age at onset in the 33 KSS patients was from 1 to 19 years based on Rowland's criteria, $22(67 \%)$ had ptosis or PEO or muscle weakness. The initial findings of the patients whose age at onset was younger than 5 years were short stature and IDDM but not ptosis or PEO. One patient whose initial finding was short stature exhibited rare renal tubular dysfunction mimicking Bartter syndrome and subsequently typical ptosis and PEO (Goto et al. 1990b). All six PS patients were younger than 2 years and did not have PEO at the time of diagnosis.

Three patients with atypical phenotype

Three patients showed the following atypical and severe clinical phenotypes: mitochondrial myopathy, encephalopathy, lactic acidosis and stroke-like episodes (MELAS), Leigh syndrome, and Reye-like syndrome.

The first patient was a 4-year-old boy. He experienced status epilepticus and syndrome of inappropriate secretion of antidiuretic hormone (SIADH) accompanied by abdominal pain and vomiting when he was 2 years old. Thereafter, he periodically had abdominal pain and vomiting once or twice a year. At 4 years and 10 months of age, he showed a stroke-like episode with disturbance of consciousness, convulsions, and severe metabolic acidosis. 
Cranial computed tomography showed a low-density area in the occipital lobes. The lactate level in serum was slightly increased $(16.5 \mathrm{mg} / \mathrm{dl}$ : normal $<15)$ and that in CSF was significantly increased $(41.7 \mathrm{mg} / \mathrm{dl})$. Muscle biopsy showed some ragged-red fibers but not strongly succinate-dehydrogenase -reactive blood vessels (SSVs). On cytochrome $c$ oxidase (COX) staining, scattered fibers with no enzyme activity were observed, suggesting focal COX deficiency. Based on these findings, we considered that MELAS was the most likely diagnosis, as neither 3243 nor 3271 mutation, which is frequently detected in MELAS patients, was found in this case.

The second patient was a 2-year-old boy. During the perinatal period, he developed pancytopenia that required treatment. His psychomotor development was within the normal range, but he showed a sudden seizure with unconsciousness and motor disturbance followed by viral gastroenteritis. Cranial magnetic resonance imaging (MRI) showed $\mathrm{T} 1$ low intensity and $\mathrm{T} 2$ high intensity in the thalamus and basal ganglia. Serum and CSF lactate levels were significantly increased ( 32 and $43 \mathrm{mg} / \mathrm{dl}$, respectively). Muscle biopsy showed no typical ragged-red fibers. On COX staining, the enzyme activity was mildly and diffusely decreased. We considered that Leigh syndrome was the most likely diagnosis for this patient.

The third patient was a 1.5-year-old boy. After an episode of vomiting and diarrhea probably due to gastroenteritis, he developed abrupt systemic edema following continuous tonic and clonic seizures. Laboratory findings showed hypoproteinemia, hypoglycemia, severe ketoacidosis with high lactate level, and liver dysfunction. MRI and organic acid and amino acid analyses showed no apparent abnormality. Liver biopsy showed small vacuoles in hepatocytes, suggesting steatosis. Muscle biopsy showed a few ragged-red fibers, and no SSVs were detected. On COX staining, fibers with no enzyme activity were occasionally observed. The patient was diagnosed as having Reye-like syndrome because he lacked specific symptoms or laboratory findings suggestive of CPEO, KSS, and PS.

Because all three patients were younger than 4 years old at the time of examination, we could not exclude the possibility that they might show other symptoms or laboratory findings indicative of CPEO, KSS, and PS in the future.

\section{Length and location of deletion}

The length and location of deletion were highly variable. The length of deletion in 135 patients ranged from 1.1 to 9.6 kilobases $(\mathrm{kb})(5.1 \pm 1.6 \mathrm{~kb}$ on average $)$. The breakpoints of the left borders were located from nt 5834 to nt 13911 and those of the right borders from nt 9519 to nt 16123, which distributed throughout the entire longer arc

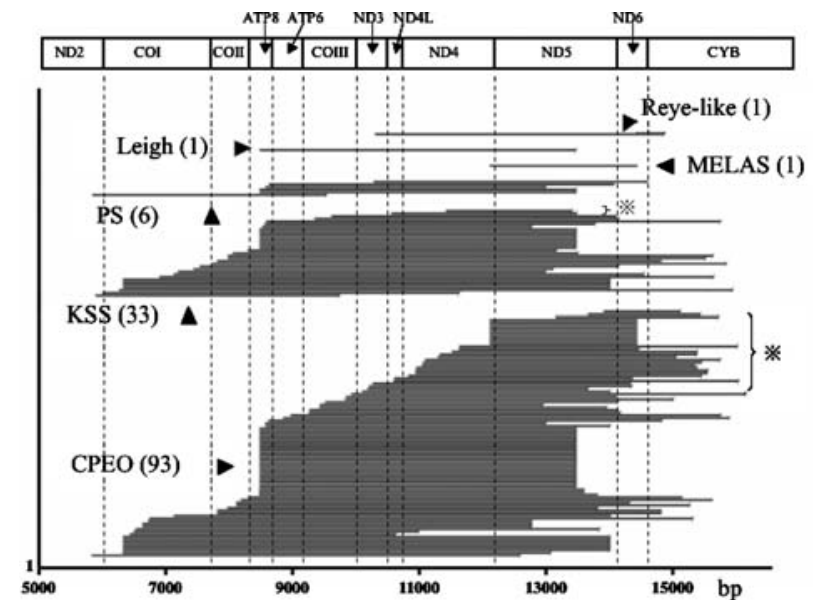

Fig. 2 Localization of 135 deletions. $N D$ complex I subunit, $C O$ complex IV subunit, ATP adenosine triphosphate synthase subunit, MELAS mitochondrial myopathy, encephalopathy, lactic acidosis, and stroke-like episodes, $※=$ patients with deletion encompassing only ND or ND plus cytochrome b (CYB) subunits

of mtDNA (Fig. 2). Only one patient had deletion within the so-called shorter arc between the two origins of mtDNA replication (nucleotide numbers 191-5730). The deletion was from nt 548 to nt 4442, including one proteincoding subunit (ND1), five complete and one incomplete transfer ribonucleic acid (tRNA) regions, and a part of the control region. The similar deletion had already been reported (Hammans et al. 1992).

We noted that the short deletions or deletions encompassing only complex I (ND) subunits might be more frequent in CPEO than in KSS (Fig. 2). To confirm this finding, we examined all patients except atypical cases (PS, MELAS, Leigh syndrome, Reye-like syndrome, and CPEO patients who have deletion at the shorter arc) regarding the relationship between age at onset and the following:

1. Length of deletion

We first examined the relationship between the length of deletion and the age at onset. The result suggests a moderate correlation (correlation coefficient $r=$ -0.51 ; Fig. 3). Furthermore, we compared the length of deletion between CPEO and KSS patients. KSS patients seemed to have significantly longer deletion than CPEO patients $(P=0.005<0.01)$.

2. Number of deleted tRNAs

Although the number of deleted tRNAs might be associated with the length of deletion, we examined the relationship between the number of deleted tRNAs and the age at onset. The result suggests a moderate correlation (correlation coefficient $r=-0.48$; Fig. 4). Furthermore, we compared the number of deleted tRNAs and the age at onset between CPEO and KSS patients. KSS patients seemed to have significantly 


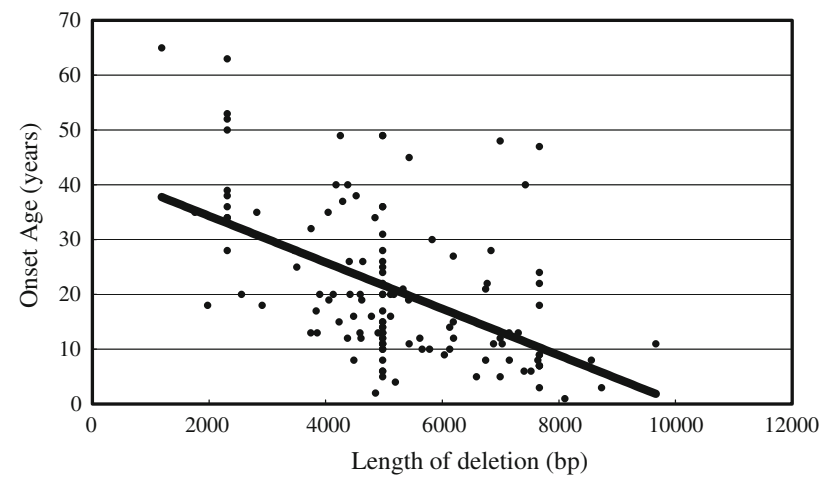

Fig. 3 Scattergram and linear regression between the length of mitochondrial DNA (mtDNA) deletion and age at onset

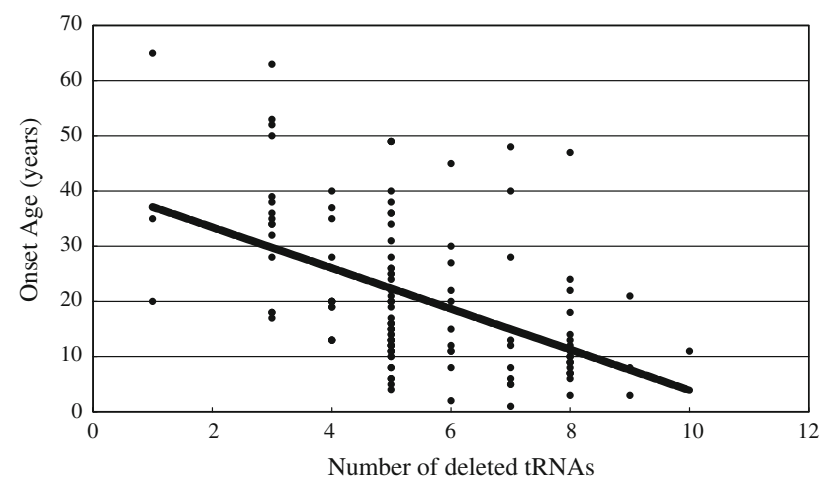

Fig. 4 Scattergram and linear regression between the number of deleted tRNAs and age at onset

more deleted tRNAs than CPEO patients $(P=0.008<0.01)$.

3. Location of deletion

The deletion sometimes included only ND subunits or ND plus cytochrome $b$ (CYB) subunits, sparing COX and adenosine triphosphate (ATP) synthase subunits (Fig. 2). To determine the relationship between the location of deletion and the age at onset, we divided the patients to group 1 (with deletion encompassing only ND or ND plus CYB subunits) and group 2 (with deletion encompassing at least one COX or ATP subunit). Group 1 consisted of $27 \%$ of the patients, whereas group 2 consisted of $73 \%$ of patients. The age at onset between groups 1 and 2 showed a statistical significance $(P<0.0001)$.

\section{Proportion of deleted mtDNA}

To assess whether heteroplasmy might play a role in phenotypic manifestation, we determined the proportion of deleted mtDNA in skeletal muscle samples from 128 patients on Southern blot analysis. It ranged from $18 \%$ to

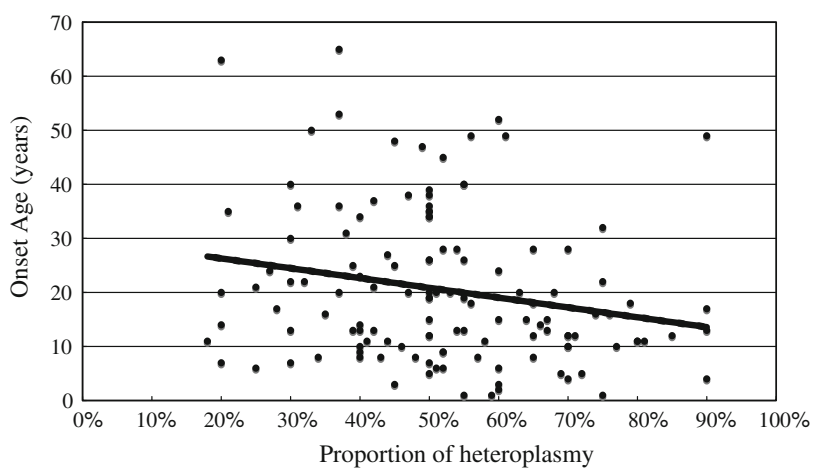

Fig. 5 Scattergram and linear regression between the proportion of deleted mitochondrial DNA (mtDNA) and age at onset

$90 \%(52.2 \pm 16.8 \%)$, but only a slight correlation between heteroplasmy and age at onset (correlation coefficient $r=-0.21$; Fig. 5) was evident. To exclude the effects of the differences in length and position as much as possible, we compared patients having the same deletion, or the socalled common 4,977-bp deletion. There were no correlations among age at onset, clinical diagnosis, clinical manifestation, and heteroplasmy (Table 2).

Sequences of deletion breakpoints

In this study, we found 81 patterns of mtDNA deletion. Direct repeats of $4 \mathrm{bp}$ or longer flanking the breakpoints were found in 96 patients (70.5\%), and those of $10 \mathrm{bp}$ or longer were found in 49 patients (36.0\%) (Table 3). It is well known that the common deletion is flanked by 13-bp direct repeats (Schon et al. 1989; Mita et al. 1990). We also found two other common deletions in addition to the most common deletion (34 patients, 25.0\%), namely, the 2,310-bp deletion from nt 12113 to nt 14421 (11 patients, 8.0\%) and the 7,664-bp deletion from nt 6330 to nt 13993 (ten patients, $7.3 \%$ ). These additional common deletions had incomplete direct repeats longer than $13 \mathrm{bp}$ with one base mismatch (Fig. 6). Note that the 2,310-bp common deletion was only detected in female patients in this study. Two previous studies describing this deletion provided no information about patient gender (Mita et al. 1990; Deugol et al. 1991).

\section{Discussion}

We examined 136 patients with single large-scale deletions in terms of their clinical phenotypes, genomic features, and their relationships. All but three patients were clinically diagnosed as having CPEO, KSS, and PS, which are widely known to be associated with mtDNA deletions. Chronologically, PS is recognized and diagnosed in infants or very young children, and the most prominent clinical feature of 
Table 2 Proportion of deletion, age at onset, clinical diagnosis, and clinical manifestations of patients with the common 4,977-bp deletion

\begin{tabular}{|c|c|c|c|c|c|c|c|c|c|c|c|}
\hline \multirow{2}{*}{$\begin{array}{l}\text { Percent } \\
\text { del }\end{array}$} & \multirow{2}{*}{$\begin{array}{l}\text { Onset } \\
\text { age } \\
\text { (years) }\end{array}$} & \multirow{2}{*}{$\begin{array}{l}\text { Clinical } \\
\text { diagnosis }\end{array}$} & \multicolumn{9}{|c|}{ Clinical manifestation } \\
\hline & & & Ptosis & PEO & Retinopathy & $\begin{array}{l}\text { Heart } \\
\text { conduction } \\
\text { block }\end{array}$ & Deafness & $\begin{array}{l}\text { Muscle } \\
\text { weakness }\end{array}$ & $\begin{array}{l}\text { Short } \\
\text { stature }\end{array}$ & Anemia & Remarks \\
\hline 20 & 20 & CPEO & O & + & - & - & - & - & - & - & \\
\hline 28 & 17 & CPEO & O & + & - & - & + & + & - & - & \\
\hline 31 & 36 & CPEO & O & + & - & - & + & + & - & - & \\
\hline 37 & 20 & CPEO & O & + & $\mathrm{U}$ & - & - & + & + & - & \\
\hline 37 & 36 & CPEO & + & + & - & - & $\bigcirc$ & + & - & - & \\
\hline 38 & 31 & CPEO & $\bigcirc$ & + & $\mathrm{U}$ & - & - & + & - & - & \\
\hline 40 & 14 & CPEO & O & + & - & - & - & + & + & - & \\
\hline 42 & 13 & CPEO & O & + & $\mathrm{U}$ & $\mathrm{U}$ & - & - & - & - & \\
\hline 45 & 25 & CPEO & O & + & $\mathrm{U}$ & - & - & - & - & - & \\
\hline 50 & 12 & CPEO & O & + & - & - & - & - & - & - & \\
\hline 50 & 15 & CPEO & O & + & $\mathrm{U}$ & - & - & + & - & - & \\
\hline 50 & 26 & CPEO & O & + & $\mathrm{U}$ & $\mathrm{U}$ & $\mathrm{U}$ & - & - & - & \\
\hline 51 & 6 & CPEO & O & + & - & - & - & - & - & - & \\
\hline 54 & 13 & CPEO & O & $\bigcirc$ & $\mathrm{U}$ & - & - & - & - & - & \\
\hline 54 & 28 & CPEO & + & $\bigcirc$ & - & + & - & + & - & - & \\
\hline 56 & 49 & CPEO & O & + & - & + & - & + & - & - & \\
\hline 60 & 24 & CPEO & O & + & $\mathrm{U}$ & $\mathrm{U}$ & $\mathrm{U}$ & + & - & - & \\
\hline 66 & 14 & CPEO & O & + & - & - & - & + & - & - & \\
\hline 70 & 10 & CPEO & O & + & + & - & - & + & - & - & \\
\hline 70 & 10 & CPEO & O & + & - & - & + & - & + & - & \\
\hline 70 & 12 & CPEO & O & + & - & - & - & + & - & - & Dysphagia \\
\hline 75 & 22 & CPEO & O & + & - & - & - & + & - & - & \\
\hline 90 & 49 & CPEO & O & + & + & - & - & + & - & - & \\
\hline 57 & 8 & KSS & O & + & + & + & - & + & + & - & IDDM + ataxia \\
\hline 58 & 11 & KSS & ○ & + & + & + & + & + & + & - & Ataxia \\
\hline 60 & 6 & KSS & + & + & + & - & + & - & ○ & - & \\
\hline 64 & 15 & KSS & ○ & + & + & + & - & + & - & - & \\
\hline 67 & 13 & KSS & $\bigcirc$ & + & + & + & - & + & - & - & \\
\hline 72 & 5 & KSS & O & + & + & - & + & + & + & - & IDDM \\
\hline 80 & 11 & KSS & O & + & + & - & + & - & + & - & Ataxia \\
\hline 81 & 11 & KSS & O & + & + & + & + & + & - & - & \\
\hline 59 & 1 & Leigh & - & - & - & - & $\mathrm{U}$ & ○ & - & + & Coma \\
\hline 55 & 1 & Pearson & - & - & $\mathrm{U}$ & $\mathrm{U}$ & $\mathrm{U}$ & $\mathrm{U}$ & - & + & Thrombocytopenia + diarrhea \\
\hline 82 & 0 days & Pearson & - & - & $\mathrm{U}$ & $\mathrm{U}$ & $\mathrm{U}$ & $\mathrm{U}$ & - & 0 & \\
\hline
\end{tabular}

Percent del percent of deleted mitochondrial DNA (mtDNA), CPEO chronic progressive external ophthalmoplegia, KSS Keans-Sayre syndrome, Leigh Leigh syndrome, Pearson Pearson's marrow pancreatic syndrome, $\bigcirc$ initial symptom, + presence of manifestation, - absence of manifestation, $U$ unknown, IDDM insulin-dependent diabetes mellitus

this syndrome is anemia. However, this anemic condition usually disappears by 1 year. There are several reports that PS patients later develop the KSS phenotype (Larsson et al. 1990; McShane et al. 1991; Akman et al. 2004). As for KSS, six out of the eight patients whose age at onset was younger than 5 years and 9 out of all 33 patients were recognized to have short stature as an initial finding. In these patients, ptosis/PEO appeared after a few years from the time when they were given medical attention by pediatricians. Thus, the possibility of mtDNA deletion disorder in such cases without a previous history of anemia must be considered.

We hypothesized that size and location of mtDNA deletion are associated with clinical features. In our previous study, evidence supporting this hypothesis was exiguous because of the small number of patients (Goto 
Table 3 Patterns of deletions and direct repeats at breakpoints

\begin{tabular}{llc}
\hline $\begin{array}{l}\text { Length of direct } \\
\text { repeats (bp) }\end{array}$ & $\begin{array}{l}\text { Patterns of } \\
\text { deletion }\end{array}$ & $\begin{array}{l}\text { Number of } \\
\text { patients }\end{array}$ \\
\hline $0-3$ & 39 & 40 \\
4 & 5 & 5 \\
5 & 7 & 16 \\
6 & 8 & 10 \\
7 & 6 & 6 \\
8 & 7 & 7 \\
9 & 3 & 3 \\
10 & 2 & 12 \\
11 & 2 & 2 \\
12 & 0 & 0 \\
13 & 2 & 35 \\
Total & 81 & 136 \\
\hline
\end{tabular}

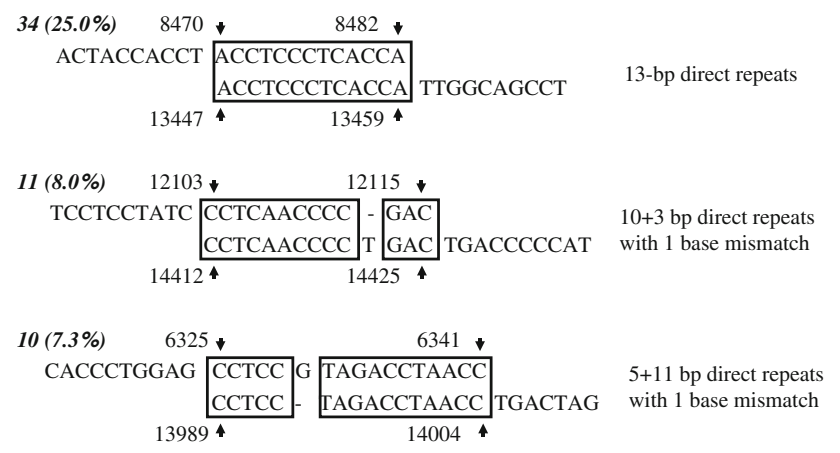

Fig. 6 Sequences at the breakpoints of three major common deletions. Direct repeats are surrounded by square, with a nucleotide number based on the standard sequence

et al. 1990a). Aure et al. (2007) divided 69 patients with mtDNA deletion to two groups according to the presence or absence of brain manifestations such as cerebellar ataxia, movement disorder, pyramidal syndrome, or dementia. They found no difference in the size and location of deletion in these two groups. In the study reported here, patients with longer deletions, in which more tRNAs were included, had earlier disease onset. Furthermore, KSS patients had longer deletion and larger number of tRNAs than CPEO patients. Taking age into account, it seems probable to assume that in patients with longer deletion, more deleted tRNAs induce the development of the KSS phenotype or enhance multisystemic involvement. We also found the patients with the deletion, including COX or ATPase subunits, showed earlier onset than those patients with the deletion limited to the region of ND and/or CYB subunits. It may support the relationship between the quality of the deletion and disease severity.

The quality of deletion is not a sole determinant of disease severity. Because patients who had deletion at the common deletion site showed various clinical symptoms (Table 2), we thought that systemic distribution and heteroplasmy (or quantity) of deletion may also be major factors causing clinical variety. The difference between KSS and CPEO is believed to be the extent of tissue or organ involvement. Although KSS is a systemic disorder and CPEO is a muscle-specific disease, these definitions are not strict. Based on Rowland's criteria of KSS, patients have muscular symptoms (ptosis, PEO, muscle weakness), retinopathy, cardiac conduction defects, and central nervous system symptoms or signs (cerebellar signs, high CSF protein concentration due to white-matter degeneration). Because our study showed that short stature and deafness were highly prevalent and IDDM and deToni-FanconiDebré syndrome were sometimes prominent manifestations of KSS, these manifestations should be added to the minor items for KSS diagnosis. All CPEO patients except one had ptosis/PEO as initial symptom. Because a substantial proportion of these patients, especially those younger than 20 years, had nonmuscular symptoms, careful assessment of systemic involvement is required in evaluating CPEO patients.

Deletions are usually heteroplasmic, and the proportion varies in each case (Moraes et al. 1989; Goto et al. 1990b) and each tissue (Marzuki et al. 1997). The previous reports and this study showed that there is no correlation between the proportion of deleted mtDNA in skeletal muscle and the age at onset. Aure et al. (2007) reported that the presence and proportion of mtDNA deletion in blood but not in skeletal muscle showed a relationship with severe prognosis in terms of rate of progression, tissular extension, and rate of survival. Because a muscle specimen was not available, we could only examine several blood samples, which were not sufficient for statistic analysis. On the other hand, the distribution of deleted mtDNA at cellular, tissue, and organ levels could not be detect by the method used. The cells with high deletion content might be lost more easily than those with low content (Hayashi et al. 1991). Regarding the relationship between the proportion of deleted mtDNA and clinical severity, the status at examination could be "the area swept by the flames" after loss of cells with high deletion content. It suggests that the remaining cells had a relatively low proportion of deletion despite the severe cell loss, which may directly induce organ symptoms such as muscle weakness. Overall, it is important to understand the mechanism of how deleted mtDNA molecules spread to the whole body or how they are limited to specific cells or tissues. Many factors may be involved in this phenomenon, such as control of replication machinery, presence of duplication, and so on.

Although slip replication (Shoffner et al. 1989) and homologous recombination have been suggested (Deugol et al. 1991), the genomic mechanism of this deletion event 
is still unknown. Direct repeats flanking breakpoints have been frequently reported. In our study, $70 \%$ of patients had 4-bp or longer direct repeats as those of previous reports (Mita et al. 1990). We emphasize that we detected two additional common deletions besides the most common deletion with 13-bp direct repeats at breakpoints (Schon et al. 1989). These new common deletions also had 13 $(10+3)$ and $16(5+11)$ bp incomplete direct repeats with a mismatch, suggesting that long direct repeats are closely associated with the deletion mechanism.

Only several deletions were found between the origin of heavy-chain replication and that of light-chain replication (from nt 191 to nt 5730), which was called a shorter arc (Moraes et al. 1991; Katayama et al. 1991; Johns and Cornblath 1991; Hammans et al. 1992; Horton et al. 1996; $\mathrm{Vu}$ et al. 2000). In our study, there was only one patient with deletion in the shorter arc. Because the same deletion was reported in two studies with three cases, this site might be a hot spot for deletion at the shorter arc. The presence of 13-bp complete direct repeats at these deletion breakpoints further suggests the importance of the length of direct repeats.

In conclusion, our data indicate that initial symptoms other than ptosis/PEO, deletion length, number of deleted tRNAs, and age at onset could be risk factors for systemic involvement of single mtDNA deletion diseases. Deletion of mtDNA has been detected in various neurodegenerative diseases such as Parkinson's disease and Alzheimer's disease (Bender et al. 2006; Kraysberg et al. 2006; Shapira 2006). Further understanding of the mechanism of deletion and its biological importance can lead to the development of a new treatment modality for a particular disease.

Acknowledgments This work was supported by grants-in-aid for scientific research on priority areas from the Ministry of Education, Science, Sports, and Culture of Japan (to YG) and by a grant of the Comprehensive Research Project on Health Sciences Focusing on Drug Innovation (KHD2207) from the Japan Health Sciences Foundation (to YG and IN). We are grateful to Ms. Kumiko Minami, Ms. Mayuko Kato, and Ms. Rika Oketa for technical assistance.

\section{References}

Akman CI, Sue CM, Shanske S, Tanji K, Bonilla E, Ojaimi J, Krishna S, Schubert R, DiMauro S (2004) Mitochondrial DNA deletion in a child with megaloblastic anemia and recurrent encephalopathy. J Child Neurol 19:258-261

Aure K, Ogier de Baulny H, Laforet P, Jardel C, Eymard B, Lombes A (2007) Chronic progressive ophthalmoplegia with large-scale mtDNA rearrangement: can we predict progression? Brain 130:1516-1524

Bender A, Krishnan KJ, Morris CM, Taylor GA, Reeve AK, Perry RH, Hersheson JS, Betts J, Klopstock T, Taylor RW, Turnbull DM (2006) High levels of mitochondrial DNA deletions in substantia nigra neurons in aging and Parkinson disease. Nat Genet 38:515-517
Campos Y, Martin MA, Caballero C, Rubio JC, de la Cruz F, Tunon T, Arenas J (2000) Single large-scale mitochondrial DNA deletion in a patient with encephalopathy, cardiomyopathy, and prominent intestinal pseudo-obstruction. Neruomuscul Disord 10:56-58

Deugol F, Nelson I, Amselem S, Romero N, Obermaier-Kusser B, Ponsot G, Marsac C, Lestienne P (1991) Different mechanisms inferred from sequences of human mitochondrial DNA deletions in ocular myopathies. Nucleic Acids Res 19:493-496

Goto Y, Koga Y, Horai S, Nonaka I (1990a) Chronic progressive external ophthalmoplegia: a correlative study of mitochondrial DNA deletions and their phenotypic expression in muscle biopsies. J Neurol Sci 100:63-69

Goto Y, Itami N, KajiiN, Tochimaru H, Endo M, Horai S (1990b) Renal tubular involvement mimicking Bartter syndrome in a patient with Kearns-Sayre syndrome. J Pediatr 116:904-910

Goto Y, Nishino I, Horai S, Nonaka I (1996) Detection of DNA fragments encompassing the deletion junction of mitochondrial genome. Biochem Biophys Res Commun 222:215-219

Hammans SR, Sweeny MG, Wicks DA, Morgan-Hughes JA, Harding AE (1992) A molecular genetic study of focal histochemical defects in mitochondrial encephalomyopathies. Brain 115:343365

Hayashi J-I, Ohta S, Kikuchi A, Takemitu M, Goto Y, Nonaka I (1991) Introduction of disease-related mitochondrial DNA deletions into HeLa cells lacking mitochondrial DNA results in mitochondrial dysfunction. Proc Natl Acad Sci USA 88:10614-10618

Holt IJ, Harding AE, Morgan-Hughes JA (1988) Deletions of muscle mitochondrial DNA in patients with mitochondrial myopathies. Nature 331:717-719

Horton TM, Petros JA, Heddi A, Shoffner J, Kaufman AE, Graham SD Jr., Gramlich T, Wallace DC (1996) Novel mitochondrial DNA deletion found in a renal cell carcinoma. Genes Chromosomes Cancer 15:95-101

Johns DR, Cornblath DR (1991) Molecular insight into the asymmetric distribution of pathogenetic human mitochondrial DNA deletions. Biochem Biophys Res Commun 174:244-250

Katayama M, Tanaka M, Yamamoto H, Ohbayashi T, Nimura Y, Ozawa T (1991) Deleted mitochondrial DNA in the skeletal muscle of aged individuals. Biochem Int 25:47-56

Kaukonen J, Juselius JK, Tiranti V, Kyttala A, Zeviani M, Comi GP, Keranen S, Peltonen L, Suomalainen A (2000) Role of adenine nucleotide translocator 1 in mtDNA maintenance. Science 289:782-785

Kraysberg Y, Kudryavtseva E, McKee AC, Geula C, Kowall NW, Khrapko K (2006) Mitochondrial DNA deletions are abundant and cause functional impairment in aged human substantia nigra neurons. Nat Genet 38:507-508

Larsson NG, Holme E, Kristiansson B, Oldfors A, Tulinius M (1990) Progressive increase of the mutated mitochondrial DNA fraction in Kearns-Sayre syndrome. Pediatr Res 28:131-136

Marzuki S, Berkovic SF, Saifuddin Noer A, Kapsa RM, Kalnins RM, Byrne E, Sasmono T, Sudoyo H (1997) Developmental genetics of deleted mtDNA in mitochondrial oculomyopathy. J Neurol Sci 145:155-162

McShane MA, Hammans SR, Sweeney M, Holt IJ, Beattie TJ, Brett EM, Harding AE (1991) Pearson syndrome and mitochondrial encephalopathy in a patient with a deletion of mtDNA. Am J Hum Genet 48:39-42

Miller SA, Dykes DD, Polesky HF (1988) A simple salting out procedure for extracting DNA from human nucleated cells. Nucleic Acids Res 16:1215

Mita S, Rizzuto R, Moraes CT, Shanske S, Arnaudo E, Fabrizi GM, Koga Y, DiMauro S, Schon EA (1990) Recombination via flanking direct repeats is a major cause of large-scale deletions of human mitochondrial DNA. Nucl Acids Res 18:561-567 
Moraes CT, DiMauro S, Zeviani M, Lombes A, Shanske S, Miranda AF, Nakase H, Bonilla E, Werneck LC, Servidei S, Nonaka I, Koga Y, Spiro AJ, Brownell KW, Schmidt B, Schotland DL, Zupanc M, Devivio DC, Schon EA, Rowland LP (1989) Mitochondrial DNA deletions in progressive external ophthalmoplegia and Kearns-Sayre syndrome. New Engl J Med 320:1293-1299

Moraes CT, Andreetta F, Bonilla E, Shanske S, DiMauro S, Schon EA (1991) Replication-competent human mitochondrial DNA lacking the heavy-strand promoter region. Mol Cell Biol 11:1631-1637

Mori K, Narahara K, Ninomiya S, Goto Y, Nonaka I (1991) Renal and skin involvement in a patient with complete Kearns-Sayre syndrome. Am J Med Genet 38:583-587

Nakai A, Goto Y, Fujisawa K, Shigematsu Y, Kikawa Y, Konishi Y, Nonaka I, Sudo M (1994) Diffuse leukodystrophy with a largescale mitochondrial DNA deletion. Lancet 343:1397-1938

Nishino I, Spinazzola A, Hirano M (1999) Thymidine phosphorylase gene mutations in MNGIE, a human mitochondrial disorder. Science 283:689-692

Ozawa T, Yoneda M, Tanaka M, Ohno K, Sato W, Suzuki H, Nishikimi M, Yamamoto M, Nonaka I, Horai S (1988) Maternal inheritance of deleted mitochondrial DNA in a family with mitochondrial myopathy. Biochem Biophys Res Commun 154:1240-1247

Poulton J, Deadman ME, Gardiner RM (1989) Duplications of mitochondrial DNA in mitochondrial myopathy. Lancet 1:236240

Rowland LP (1983) Molecular genetics, pseudogenetics, and clinical neurology. Neurology 33:1179

Rötig A, Colonna M, Bonnefont JP, Blanche S, Fischer A, Saudubray JM, Munnich A (1989) Mitochondrial DNA deletion in Pearson's marrow/pancreas syndrome. Lancet 1:902-903

Schon EA, Rizzuto R, Moraes CT, Nakase H, Zeviani M, DiMauro S (1989) A direct repeat is a hot spot for large-scale deletions of human mitochondrial DNA mutations. Science 244:346-349
Servidei S, Zeviani M, Manfredi G, Ricci E, Silvestri G, Bertini E, Gellera C, Di Mauro S, Di Donato S, Tonali P (1991) Dominantly inherited mitochondrial myopathy with multiple deletions of mitochondrial DNA: clinical, morphologic, and biochemical studies. Neurology 41:1053-1059

Shapira AHV (2006) Mitochondrial disease. Lancet 368:70-82

Shoffner JM, Lott MT, Voljavec AS, Soueidan SA, Costigan DA, Wallace DC (1989) Spontaneous Kearns-Sayre/chronic external ophthalmoplegia plus syndrome associated with a mitochondrial DNA deletion: a slip-replication model and metabolic therapy. Proc Natl Acad Sci USA 86:7952-7956

Spelbrink JN, Li FY, Tiranti V, Nikali K, Yuan QP, Tariq M, Wanrooij S, Garrido N, Comi G, Morandi L, Santoro L, Toscano A, Fabrizi GM, Somer H, Croxen R, Beeson D, Poulton J, Suomalainen A, Jacobs HT, Zeviani M, Larsson C (2001) Human mitochondrial DNA deletions associated with mutations in the gene encoding Twinkle, a phage $\mathrm{T} 7$ gene 4-like protein localized in mitochondria. Nat Genet 28:223-231

Van Goethem G, Dermaut B, Lofgren A, Martin JJ, Van Broeckhoven C (2001) Mutation of POLG is associated with progressive external opthalmoplegia characterized by mtDNA deletions. Nat Genet 28:211-212

Vu TH, Tanji K, Pallotti F, Golzi V, Hirano M, DiMauro S, Bonilla E (2000) Analysis of mtDNA deletions in muscle by in situ hybridization. Muscle Nerve 23:80-85

Zeviani M, Moraes CT, DiMauro S, Nakase H, Bonilla E, Schon EA, Rowland LP (1988) Deletions of mitochondrial DNA in KearnsSayre syndrome. Neurology 38:1339-1346

Zeviani M, Servidei S, Gellera C, Bertini E, DiMauro S, DiDonato S (1989) An autosomal dominant disorder with multiple deletions of mitochondrial DNA starting at the D-loop region. Nature 339:309-311 